\title{
Changes in Central Macular Thickness following Single Session Multispot Panretinal Photocoagulation
}

\author{
Nawat Watanachai, Janejit Choovuthayakorn, Direk Patikulsila, and Nimitr Ittipunkul \\ Department of Ophthalmology, Faculty of Medicine, Chiang Mai University, Chiang Mai 50200, Thailand \\ Correspondence should be addressed to Janejit Choovuthayakorn; janejit.c@cmu.ac.th
}

Received 14 October 2014; Revised 26 December 2014; Accepted 6 January 2015

Academic Editor: Lawrence S. Morse

Copyright ( 2015 Nawat Watanachai et al. This is an open access article distributed under the Creative Commons Attribution License, which permits unrestricted use, distribution, and reproduction in any medium, provided the original work is properly cited.

\begin{abstract}
Purpose. To determine changes in central subfield (CSF) macular thickness and best corrected visual acuity (BCVA) following single session, multispot panretinal photocoagulation (PRP). Methods. Forty eyes of 33 patients with newly diagnosed proliferative diabetic retinopathy were treated with single session, 20-millisecond, multispot PRP. Changes in central macular thickness and BCVA at 4- and 12-week follow-up were compared to baseline measurements. Results. Each eye received a mean (SD) of 2,750 (686.7) laser spots. At 4 -week follow-up, there was a statistically significant $24.0 \mu \mathrm{m}$ increase in mean CSF thickness $(P=0.001)$, with a $17.4 \mu \mathrm{m}$ increase from baseline at 12 -week follow-up $(P=0.002)$. Mean logMAR BCVA increased by $0.05 \operatorname{logMAR}$ units $(P=0.03)$ at 4 week follow-up. At 12-week follow-up, BCVA had almost returned to normal with only an increase of 0.02 logMAR units compared to baseline $(P=0.39)$. Macular edema occurred in 2 eyes $(5 \%)$ at 12 -week follow-up. Conclusions. Macular thickening occurs following single session, 20-millisecond, multispot PRP, with a corresponding, mild change in BCVA. However, the incidence of macular edema appears to be low in these patients. Single session, 20-millisecond, multispot PRP appears to be a safe treatment for patients with proliferative diabetic retinopathy.
\end{abstract}

\section{Introduction}

Panretinal photocoagulation (PRP) is performed as a standard treatment for proliferative diabetic retinopathy (PDR) and is also frequently used to treat severe nonproliferative diabetic retinopathy. Its utility in reducing the risk of severe vision loss was demonstrated by the Diabetic Retinopathy Study and the Early Treatment Diabetic Retinopathy Study (ETDRS) [1-3]. The pathophysiologic changes leading to neovascularization regression following PRP remain uncertain. However, it is postulated that PRP decreases the metabolic activity of the ischemic retina, reduces the production of angiogenic factors, and improves inner retinal circulation [4]. Conventional PRP is delivered in one or more sessions in single spot mode, with the laser spot size ranging from 100 to $500 \mu \mathrm{m}$ and a pulse duration ranging from 100 to $200 \mathrm{mil}-$ liseconds (ms). While the overall visual benefit of this therapy is not disputed, visual disabilities have been reported after conventional PRP, including central visual loss caused by the development of macular edema and peripheral visual loss from extensive, expanding inner retinal scarring [5-7].

In an attempt to minimize the drawbacks of conventional PRP, an innovative photocoagulator with the capability to deliver multispot, medium pulse duration laser burns was recently introduced into clinical practice [8]. Optimal laser parameters applied with $20 \mathrm{~ms}$ pulse duration PRP have been investigated at several centers [9-11]. Several studies have demonstrated comparable efficacy of multispot, $20 \mathrm{~ms}$ pulse duration PRP, delivered in either a single session or multiple sessions, to conventional, single spot $100 \mathrm{~ms}$ pulse duration PRP, in terms of regression of retinal neovascularization [12, 13]. In addition, $20 \mathrm{~ms}$ pulse duration PRP resulted in more uniformity of laser spots, less inner retinal tissue damage, and increased patient comfort compared to conventional $100 \mathrm{~ms}$ pulse duration PRP [14-18]. Single session, multispot, $20 \mathrm{~ms}$ pulse duration PRP is well tolerated by patients in part because it is less time-consuming and reportedly less painful. In addition, single session PRP reduces both direct 
and indirect hospital visit expenses for patients while simultaneously improving clinic efficiency for ophthalmologists. For these reasons, single session, multispot, $20 \mathrm{~ms}$ pulse duration PRP is increasingly being performed in clinical practice. However, the adverse effects of single session, multispot, $20 \mathrm{~ms}$ pulse duration PRP, including postlaser macular edema, need to be addressed. Unlike stereoscopic biomicroscopy, optical coherence tomography (OCT) is able to quantitatively evaluate subtle changes in macular thickness $[19,20]$ and has been used for objective assessment of macular thickness changes in both clinical practice and in numerous clinical trials.

The primary aim of this study is to evaluate the effect of single session, multispot, $20 \mathrm{~ms}$ pulse duration PRP on central macular thickness, as measured by spectral domain optical coherence tomography (SD-OCT). In addition, best corrected visual acuity (BCVA) changes and other complications related to PRP are reported to provide additional insight into this laser therapy delivery system.

\section{Materials and Methods}

The medical records of 35 patients presenting to the Department of Ophthalmology, Faculty of Medicine, Chiang Mai University, Thailand, from January 2012 to January 2013, with newly diagnosed, treatment naïve PDR, were retrospectively reviewed. The study was conducted according to the tenets of the Declaration of Helsinki. The hospital institutional review board and ethics committee approved the study protocol.

2.1. Patient Eligibility. Patients with type 1 or type 2 diabetes and newly diagnosed PDR were enrolled if they met the following inclusion criteria: (1) age $\geq 18$ years; (2) BCVA of 70 ETDRS letters or better (Snellen equivalent of 6/12 or better); (3) central subfield (CSF) retinal thickness measured by SDOCT $\leq 320 \mu \mathrm{m}$ with no structural abnormalities. CSF was defined as a circular area centered around the fovea with a diameter of 1,000 $\mu \mathrm{m}$. Exclusion criteria included the following: (1) history of prior treatments for diabetic macular edema (DME); (2) history of prior PRP; (3) history of intraocular surgery within the last 6 months; (4) presence of subretinal or intraretinal fluid or cystic changes on OCT in the CSF; (5) aphakia; (6) coexisting ocular diseases that could influence VA and macular thickness; (7) chronic renal failure requiring dialysis or kidney transplant; (8) systemic blood pressure (BP) more than 180/110 $\mathrm{mmHg}$; (9) inadequate media clarity to perform complete laser in one session; (10) inadequate follow-up, defined as missing the 4- or 12-week follow-up visits.

2.2. Study Design. Patients' baseline characteristics were recorded including age, sex, type and duration of diabetes, glycosylated hemoglobin A1C level within the last 3 months, and disease laterality. All patients underwent a complete ophthalmic examination including BCVA, intraocular pressure (IOP) measurement using Goldmann applanation tonometer, slit-lamp biomicroscopy, and dilated fundus examination. Color fundus photography was performed with a fundus camera (KOWA VX-10i, Kowa, Tokyo, Japan) and retinal thickness measurement was performed with a SD-OCT (Spectralis; Heidelberg Engineering, CA, USA). The OCT image was scanned in a $20 \times 20$-degree high resolution volumetric pattern consisting of 49 horizontal B-scan lines with 1,024 A-scans per line. All scans were reviewed for accuracy by one investigator (Janejit Choovuthayakorn). The patients underwent follow-up at 4 weeks and 12 weeks $( \pm 7$ days) after PRP. BCVA, IOP, slit-lamp biomicroscopy, fundus photography, and OCT imaging were performed at each follow-up visit. One masked investigator (Direk Patikulsila) reviewed the fundus photographs, comparing baseline and subsequent follow-up photos to assess for the regression of neovascularization.

2.3. Laser Photocoagulation Technique. PRP was performed with a $532 \mathrm{~nm}$ frequency-doubled neodymium-doped yttrium aluminium garnet (Nd-YAG) solid-state pattern scan laser (VALON pattern laser, Dual Laser Ltd. Oy, Vantaa, Finland) using a SuperQuad 160 contact lens (laser spot magnification of 2.0; Volk Optical Inc., Mentor, OH). A $4 \times 4$ multispot array with $200 \mu \mathrm{m}$ spot size, $20 \mathrm{~ms}$ pulse duration, and 1.5-width spot spacing was used. The burn intensity was titrated until a gray-white opacity was achieved. PRP was placed from just outside the vascular arcades to the peripheral retina, with care taken to prevent laser burns from encroaching within 2 disc diameters (DD) temporal to fovea or $1 \mathrm{DD}$ nasal to the optic disc. All laser sessions were delivered under topical anesthesia ( $0.5 \%$ tetracaine hydrochloride ophthalmic solution) by one retinal specialist (Nawat Watanachai).

2.4. Statistical Analysis. Descriptive statistics were performed for patients' baseline characteristics and reported as mean (standard deviation, SD) or median (range) for continuous data and frequency (percentage) for category data. A paired $t$ test was used to compare the mean CSF thickness and BCVA in eyes at baseline and at each follow-up visit. Pearson's correlation was performed to evaluate the correlation of changes in CSF thickness and BCVA at each follow-up visit. Univariate logistic regression was used to evaluate the associations between changes in CSF thickness at 12-week follow-up and other parameters. BCVA was converted to a logarithm of the minimal angle of resolution (logMAR) units for statistical calculation. All analyses were based on a 2-tailed test of significance and performed by Stata version 12.0 (College Station, TX; StataCorp LP). A $P<0.05$ was considered significant.

\section{Results}

Of the 35 patients who met all the eligibility criteria, 2 patients were excluded due to inadequate follow-up. Forty eyes of 33 consecutive patients were reviewed. The mean age (SD) of the patients was 52.3 (7.7) years. Of the 33 patients, 32 (97\%) had type 2 diabetes and one (3\%) had type 1 diabetes. Patients' baseline characteristics are shown in Table 1. For laser application, the mean total number (SD) of spots was 2,750 (686.7) with a mean laser power (SD) of $399(116.8) \mathrm{mW}$ delivered per eye. The laser parameters are shown in Table 2.

The mean CSF thickness (SD) at baseline was 274.3 (24.9) $\mu \mathrm{m}$. At 4-week follow-up, the mean CSF thickness 
TABLE 1: Baseline characteristics of patients undergoing single session, multispot, 20-millisecond, panretinal photocoagulation.

\begin{tabular}{lc}
\hline Characteristics & \\
\hline Gender, number (\%) & $11(33.3)$ \\
$\quad$ Male & $22(66.7)$ \\
$\quad$ Female & $52.3(7.7)$ \\
Age (year), mean (SD) & \\
Laterality, number (\%) & $22(55.0)$ \\
$\quad$ Right & $18(45.0)$ \\
$\quad$ Left & \\
Type of diabetes, $n(\%)$ & $1(3.0)$ \\
$\quad$ I & $32(97.0)$ \\
$\quad$ II & $10(0.2-25.0)$ \\
Duration of diabetes (year), median (min-max) & $9.2(2.4)$ \\
Glycosylated HbA1C (mg/dL), mean (SD) & $0.13(0.11)$ \\
Baseline logMAR BCVA, mean (SD)
\end{tabular}

SD: standard deviation, logMAR: logarithm of the minimal angle of resolution visual acuity.

TABLE 2: Laser parameters performed in single session, multispot, 20-millisecond panretinal photocoagulation.

\begin{tabular}{lc}
\hline Parameters & \\
\hline Laser power $(\mathrm{mW})$, mean $(\mathrm{SD})$ & $399(116.8)$ \\
Laser fluence $\left(\mathrm{J} / \mathrm{cm}^{2}\right)$, median (range) & $5.4(4.7$ to 36.9$)$ \\
Total laser area $\left(\mathrm{mm}^{2}\right)$, mean $(\mathrm{SD})$ & $402.0(155.8)$ \\
Total laser energy, mean (SD) & $21.7(8.0)$ \\
Total laser burn (spots), mean (SD) & $2,750.0(686.7)$ \\
Duration of treatment (min), mean (SD) & $4.9(0.9)$ \\
\hline
\end{tabular}

mW: milliwatt, SD: standard deviation, J: Joule, and min: minute.

increased by $24.0 \mu \mathrm{m}$ (95\% CI; 12.8 to $35.1 \mu \mathrm{m}, P=0.001)$ and at 12 -week follow-up the CSF thickness increased by $17.4 \mu \mathrm{m}$ (95\% CI; 6.2 to $28.6 \mu \mathrm{m}, P=0.002$ ). Mean CSF thickness at baseline and at given follow-up visits is shown in Figure 1. The mean BCVA (SD) at baseline was 0.13 (0.11) logMAR units. At 4-week follow-up, there was a statistically significant increase in mean BCVA to 0.18 logMAR units (95\% CI of mean difference; 0.03 to $0.10 \log$ MAR units, $P=0.03$ ). However, at 12-week follow-up, the increase in mean BCVA was no longer statistically significant at $0.14 \log$ MAR units (95\% CI of mean difference; -0.01 to $0.03 \log$ MAR units, $P=0.34$ ). Changes in CSF thickness and BCVA at 4-week follow-up (Pearson's correlation coefficient: $-0.58, P=0.001$ ) and at 12-week follow-up (Pearson's correlation coefficient: -0.31 , $P=0.06$ ) were inversely correlated; however, the correlations were minimal. The changes in BCVA did not well correlate with the degree of CSF thickness changes. No severe visual loss occurred in this study.

At 12-week follow-up, univariate analysis showed that age, baseline VA, CSF thickness, glycosylated HbA1C level, and laser parameters were not significantly associated with changes in CSF thickness (Table 3). Two of the 40 eyes (5\%) developed clinically significant macular edema requiring treatment. Additional PRP was performed on 10 eyes (25\%).

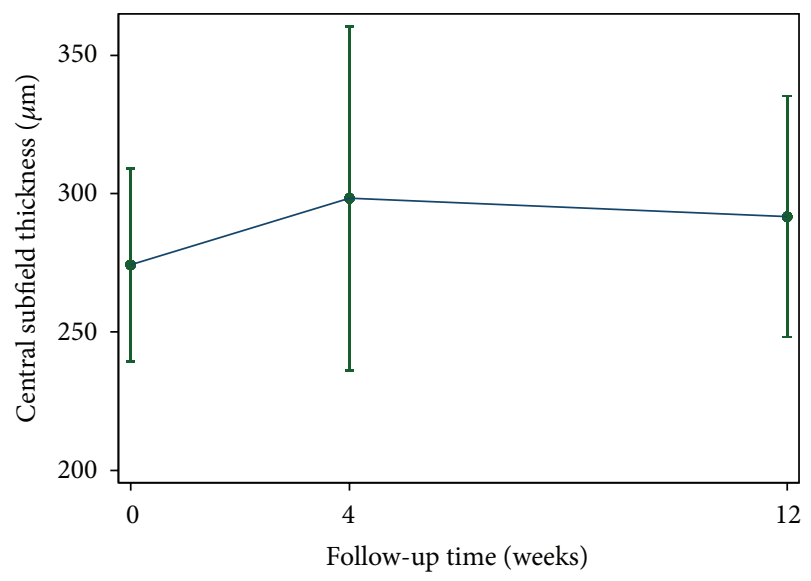

FIGURE 1: Changes in macular central subfield thickness after single session, multispot, 20-millisecond panretinal photocoagulation.

No patient experienced exudative retinal detachment, choroidal detachment, or severe visual loss.

\section{Discussion}

The present study evaluated the effect of single session, multispot, $20 \mathrm{~ms}$ pulse duration PRP on CSF thickness and BCVA in patients with newly diagnosed PDR without centerinvolving macular edema as confirmed by OCT. In this cohort, there was a statistically significant increase in CSF thickness at both the 4- and 12-week follow-up, while BCVA returned close to baseline at the 12 -week follow-up visit. However, macular edema requiring further treatment was noted in $5 \%$ of treated eyes at the 12 -week visit. There were no other adverse effects related to PRP.

Conventional PRP is the standard treatment for patients with PDR, with well-documented evidence of reduction in severe visual loss $[1,3]$. Nevertheless, visually significant macular edema following PRP has been noted on slit-lamp biomicroscopy [21]. The introduction and application of OCT in the clinical setting have allowed for the detection and quantification of subtle macular changes [20]. Macular OCT assessment following PRP has been subsequently studied $[6,22-$ 24]. Those studies showed significant, mild macular thickening compared to baseline occurring after one or more sessions of conventional PRP. In these studies, macular edema either resolved or persisted throughout follow-up (ranging from 16 weeks to 12 months). However, an increase in macular thickness resulting in macular edema was reported in $9.7 \%$ to $15.7 \%$ of treated eyes, with follow-up ranging from 12 to 34 weeks. Pattern scan laser photocoagulators capable of delivering multispot arrays of laser at a reduced pulse duration of $20 \mathrm{~ms}$ have recently been introduced into clinical practice. The benefits of this laser delivery system include decreased time required to deliver treatment and improved patient comfort compared to conventional PRP $[25,26]$. Consequently, many providers prefer multispot, $20 \mathrm{~ms}$ PRP to treat PDR. However, the effect of multispot, $20 \mathrm{~ms}$ PRP on macular thickness has been raised as a potential area of concern. 
TABLE 3: Univariate analysis of factors related to changes incentral subfield thickness at 12-week follow-up after single session, multispot, 20-millisecond panretinal photocoagulation.

\begin{tabular}{|c|c|c|c|}
\hline \multirow{2}{*}{$\begin{array}{l}\text { Characteristics } \\
\text { mean (SD) }\end{array}$} & \multicolumn{2}{|c|}{ Changes in CSF thickness } & \multirow{2}{*}{$P$ value } \\
\hline & $\geq 12 \mu \mathrm{m}$ & $<12 \mu \mathrm{m}$ & \\
\hline Gender, $n(\%)$ & & & 0.02 \\
\hline Male & $3(21.4)$ & $11(78.8)$ & \\
\hline Female & $16(61.5)$ & $10(38.5)$ & \\
\hline Age, year & $52.58(7.9)$ & $52.0(7.7)$ & 0.82 \\
\hline Baseline logMAR BCVA, mean (SD) & $0.13(0.08)$ & $0.14(0.09)$ & 0.57 \\
\hline Baseline CSF thickness, $\mu \mathrm{m}$ & $275.5(36.5)$ & $273.2(34.3)$ & 0.83 \\
\hline Baseline $\mathrm{HbAlC}, \mathrm{mg} / \mathrm{dL}$ & $8.7(2.2)$ & $9.7(2.4)$ & 0.16 \\
\hline \multicolumn{4}{|l|}{ PRP parameters } \\
\hline Laser power $(\mathrm{mW})$ & $380.0(110.2)$ & $420.0(122.7)$ & 0.36 \\
\hline Laser fluence $\left(\mathrm{J} / \mathrm{cm}^{2}\right)$ & $5.4(3.7)$ & $5.4(3.2)$ & 0.84 \\
\hline Laser area $\left(\mathrm{mm}^{2}\right)$ & $369.4(149.7)$ & $431.4(158.9)$ & 0.21 \\
\hline Total laser energy & $21.7(7.2)$ & $21.7(8.8)$ & 0.87 \\
\hline Total laser burn (spot) & $2889.0(776.1)$ & $2625.0(585.3)$ & 0.78 \\
\hline Duration of PRP (min) & $5.1(0.9)$ & $4.7(0.7)$ & 0.18 \\
\hline
\end{tabular}

SD: standard deviation, VA: visual acuity, CSF: central subfield, mW: milliwatt, J: Joule, min: minute, and PRP: pan retinal photocoagulation.

In the Manchester Pascal Study, Muqit et al. [14] conducted a randomized study comparing single spot, $100 \mathrm{~ms}$, multisession PRP with a multispot, $20 \mathrm{~ms}$ single session PRP. In their cohort, 19 eyes received multispot, $20 \mathrm{~ms}$, single session PRP. These patients developed a statistically insignificant $2 \mu \mathrm{m}$ increase in CSF thickness at 4-week posttreatment, with a statistically insignificant $2 \mu \mathrm{m}$ decrease at 12 -week posttreatment. Macular edema was not observed in either arm of their study. However, the total number of laser spots performed in the eyes receiving multispot PRP was 1,500, comparable to the conventional PRP arm. Several studies have suggested that 1,500 laser spots may be insufficient for multispot, $20 \mathrm{~ms}$ PRP. Chappelow et al. [27] retrospectively studied 82 eyes with newly diagnosed, high-risk PDR who had at least 6 months of follow-up. They reported that eyes treated with multispot, $20 \mathrm{~ms}$ PRP exhibited a higher treatment failure rate, defined as either persistence or recurrence of neovascularization, than conventional PRP when delivered as a comparable number of laser spots. They hypothesized that the higher laser fluence of conventional, $100 \mathrm{~ms}$ PRP led to a larger area of heat diffusion and a larger area of coagulated retina following $100 \mathrm{~ms}$ conventional PRP, accounting for the difference in efficacy between these two laser parameters. Other studies have also shown that higher numbers of laser spots are required for multispot, $20 \mathrm{~ms}$ PRP to obtain a similar total retinal treatment area as that seen with $100 \mathrm{~ms}$ PRP $[11,13]$.

The present study showed a statistically significant increase in CSF thickness of $24 \mu \mathrm{m}$ and $17 \mu \mathrm{m}$ at $4-$ and 12week follow-up, respectively, with an average of 2,750 laser spots delivered. The higher number of laser spots in the present study may explain the increased CSF thickening observed. Oh et al. [28] retrospectively reviewed 129 eyes after single session, multispot, $20 \mathrm{~ms}$ PRP. With a mean number of 3,125 laser spots delivered, they reported a statistically significant CSF thickness increase of $20 \mu \mathrm{m}$ at 1-month follow-up. At 12-week follow-up, $8.5 \%$ of the eyes in their study developed macular edema. In the present study, $5 \%$ of the eyes were noted to develop macular edema. Oh and colleagues used multivariate analysis to study risk factors for the development of macular edema following PRP. They found that a thickened macula and the presence of intraretinal cystoid spaces or subretinal fluid on baseline OCT were significant predictors for macular edema development following PRP. However, no patients in the present study had these features on pretreatment OCT, which may explain the slightly lower incidence of macular edema in this study. Our results confirm that careful posttreatment monitoring is required to detect the development of macular edema following multispot PRP, regardless of the presence of preexisting risk factors for the development of macular edema.

The effect of single-spot, $20 \mathrm{~ms}$ pulse duration PRP on macular edema has also been investigated. Mirshahi et al. [26] carried out a randomized study comparing single spot, $20 \mathrm{~ms}$ PRP with single spot, $100 \mathrm{~ms}$ PRP in 66 eyes. In the $20 \mathrm{~ms}$ PRP arm, with a mean of 2,125 laser spots, they reported a $13 \mu \mathrm{m}$ increase in CSF thickness at 1 -month and $9 \mu \mathrm{m}$ increase at 4 month follow-up compared to baseline. The group receiving conventional, $100 \mathrm{~ms}$ PRP had a $53 \mu \mathrm{m}$ increase in CSF thickness at 1-month and a $50.8 \mu \mathrm{m}$ increase at 4-month follow-up compared to baseline, with a mean of 1,218 laser spots. Mirshahi and colleagues showed increasing post-PRP macular thickening in the single spot, $100 \mathrm{~ms}$ PRP group. Post-PRP macular thickening in the single spot, 20 ms PRP groups was similar to the present study; however, more time was required to deliver treatment in their cohort since they utilized single 
spot therapy. This data, combined with the findings of the present study, suggests that $20 \mathrm{~ms}$ PRP, whether delivered as multispot or single spot treatment, reduces CSF thickness following PRP. Multispot treatment has the advantage of reducing treatment time, though single spot, $20 \mathrm{~ms}$ treatment may be a good option for physicians who do not have access to a multispot laser system.

Previous studies have demonstrated that the range of repeatability and reproducibility of SD-OCT CSF thickness measurements in patients with diabetic macular edema is 8 to $12 \mu \mathrm{m}$ [29-31]. We performed a univariate analysis for CSF macular changes beyond $12 \mu \mathrm{m}$ to evaluate factors related to macular thickness changes. Only gender was found to correlate with increased CSF thickness. However, the sample size was not large enough to calculate a power association. Further study of factors associated with post-PRP macular edema development is needed.

With regards to the effect of PRP on BCVA, Muqit et al. [14] reported stabilized vision over 12-week follow-up in patients who received 1,500-spot, single session, multispot, $20 \mathrm{~ms}$ PRP. Oh et al. reported a significant increase in mean logMAR BCVA from 0.2 at baseline to 0.24 at both 1- and 3month visits [28]. The present study shows a trend toward a statistically significant increase in mean logMAR BCVA from 0.13 to 0.18 at 4 -week follow-up compared to baseline. BCVA then improved to $0.14 \log$ MAR units at 12-week follow-up. The change in BCVA from baseline to 12-week follow-up was not statistically significant. A transient increase in logMAR BCVA may occur in the early phase following multispot, $20 \mathrm{~ms}$, single session PRP, and subsequent improvement in BCVA to baseline level may be observed at 12-week followup. However, while the increase in logMAR BCVA at 4-week follow-up was statistically significant, it represented less than 1 line of vision and is likely not clinically meaningful.

This study is not without limitations, including those inherent to a retrospective study. Our findings, including the decreased incidence of macular edema compared to previous studies, may have been influenced by the small sample size. However, it is equally plausible that the decreased incidence of macular edema was secondary to the absence of prePRP risk factors for macular edema. Total follow-up for this study was short; however, it mirrors common clinical practice and as such provides valuable prognostic information to providers. When possible, our results were compared to those of other studies, though direct comparisons were limited by variation in study design.

In conclusion, patients treated with multispot, $20 \mathrm{~ms}$ PRP in a single session were shown to have slight macular thickening that persisted through 12 -week follow-up. Two patients (5\%) developed macular edema, which is slightly less common than previous reports. In addition, logMAR BCVA increased slightly at 4-week follow-up, improving to near baseline at 12-week follow-up. This study adds additional support to the use of single session, multispot, $20 \mathrm{~ms}$ PRP in common clinical practice. While rare, care should be taken in monitoring patients for the development of macular edema following single session, multispot PRP, as this can be vision threatening.

\section{Conflict of Interests}

The authors have no proprietary or commercial interest in any materials discussed in this paper. The authors have no conflict of interests, including financial interest, related to this paper.

\section{Acknowledgments}

All the authors would like to thank Duanpen Narongjunchai and Soontaree Aupapong, registered nurses, for their assistance with fundus photography and OCT imaging. This study received funding support from the Faculty of Medicine, Chiang Mai University, Thailand.

\section{References}

[1] “The Diabetic Retinopathy Study Research Group. Preliminary report on effects of photocoagulation therapy," American Journal of Ophthalmology., vol. 81, no. 4, pp. 383-369, 1976.

[2] The Diabetic Retinopathy Study Research Group, "Indications for photocoagulation treatment of diabetic retinopathy: Diabetic Retinopathy Study report no. 14," International Ophthalmology Clinics, vol. 27, no. 4, pp. 239-253, 1987.

[3] Early Treatment Diabetic Retinopathy Study Research Group, "Early photocoagulation for diabetic retinopathy. ETDRS report no. 9," Ophthalomology, vol. 98, no. 5, supplement, pp. 766-785, 1991.

[4] J. Spranger, H.-P. Hammes, K. T. Preissner, H. Schatz, and A. F. H. Pfeiffer, "Release of the angiogenesis inhibitor angiostatin in patients with proliferative diabetic retinopathy: association with retinal photocoagulation," Diabetologia, vol. 43, no. 11, pp. 1404-1407, 2000.

[5] M. Shimura, K. Yasuda, T. Nakazawa, and M. Tamai, "Visual dysfunction after panretinal photocoagulation in patients with severe diabetic retinopathy and good vision," American Journal of Ophthalmology, vol. 140, no. 1, pp. 8.el-8.e10, 2005.

[6] M. Soman, S. Ganekal, U. Nair, and K. G. R. Nair, "Effect of panretinal photocoagulation on macular morphology and thickness in eyes with proliferative diabetic retinopathy without clinically significant macular edema," Clinical Ophthalmology, vol. 6, no. 1, pp. 2013-2017, 2012.

[7] A. Nonaka, J. Kiryu, A. Tsujikawa et al., "Inflammatory response after scatter laser photocoagulation in nonphotocoagulated retina," Investigative Ophthalmology and Visual Science, vol. 43, no. 4, pp. 1204-1209, 2002.

[8] M. S. Blumenkranz, D. Yellachich, D. E. Andersen et al., "Semiautomated patterned scanning laser for retinal photocoagulation," Retina, vol. 26, no. 3, pp. 370-376, 2006.

[9] A. Jain, M. S. Blumenkranz, Y. Paulus et al., "Effect of pulse duration on size and character of the lesion in retinal photocoagulation," Archives of Ophthalmology, vol. 126, no. 1, pp. 78-85, 2008.

[10] M. M. K. Muqit, J. C. B. Gray, G. R. Marcellino et al., "In vivo laser-tissue interactions and healing responses from 20-vs 100millisecond pulse pascal photocoagulation burns," Archives of Ophthalmology, vol. 128, no. 4, pp. 448-455, 2010.

[11] D. Palanker, D. Lavinsky, M. S. Blumenkranz, and G. Marcellino, "The impact of pulse duration and burn grade on size of retinal photocoagulation lesion: implications for pattern density," Retina, vol. 31, no. 8, pp. 1664-1669, 2011. 
[12] A. G. Salman, "Pascal laser versus conventional laser for treatment of diabetic retinopathy," Saudi Journal of Ophthalmology, vol. 25, no. 2, pp. 175-179, 2011.

[13] P. Muraly, P. Limbad, K. Srinivasan, and K. Ramasamy, "Single session of pascal versus multiple sessions of conventional laser for panretinal photocoagulation in proliferative diabetic retinopathy: a comparitive study," Retina, vol. 31, no. 7, pp. 13591365, 2011.

[14] M. M. K. Muqit, G. R. Marcellino, D. B. Henson et al., "Singlesession vs multiple-session pattern scanning laser panretinal photocoagulation in proliferative diabetic retinopathy: the Manchester Pascal study," Archives of Ophthalmology, vol. 128, no. 5, pp. 525-533, 2010.

[15] C. Sanghvi, R. McLauchlan, C. Delgado et al., "Initial experience with the Pascal photocoagulator: a pilot study of 75 procedures," British Journal of Ophthalmology, vol. 92, no. 8, pp. 1061-1064, 2008.

[16] M. Nagpal, S. Marlecha, and K. Nagpal, "Comparison of laser photocoagulation for diabetic retinopathy using 532-nm standard laser versus multispot pattern scan laser," Retina, vol. 30, no. 3, pp. 452-458, 2010.

[17] S. Sheth, P. Lanzetta, D. Veritti, I. Zucchiatti, C. Savorgnani, and F. Bandello, "Experience with the Pascal photocoagulator: an analysis of over 1200 laser procedures with regard to parameter refinement," Indian Journal of Ophthalmology, vol. 59, no. 2, pp. 87-91, 2011.

[18] D. Modi, P. Chiranand, and L. Akduman, "Efficacy of patterned scan laser in treatment of macular edema and retinal neovascularization," Clinical Ophthalmology, vol. 3, no. 1, pp. 465-470, 2009.

[19] D. J. Browning, M. D. McOwen, R. M. Bowen Jr., and T. L. O’Marah, "Comparison of the clinical diagnosis of diabetic macular edema with diagnosis by optical coherence tomography," Ophthalmology, vol. 111, no. 4, pp. 712-715, 2004.

[20] J. C. Brown, S. D. Solomon, S. B. Bressler, A. P. Schachat, C. DiBernardo, and N. M. Bressler, "Detection of diabetic foveal edema: contact lens biomicroscopy compared with optical coherence tomography," Archives of Ophthalmology, vol. 122, no. 3, pp. 330-335, 2004.

[21] H. R. McDonald and H. Schatz, "Visual loss following panretinal photocoagulation for proliferative diabetic retinopathy," Ophthalmology, vol. 92, no. 3, pp. 388-393, 1985.

[22] A. J. Brucker, H. Qin, A. N. Antoszyk et al., "Observational study of the development of diabetic macular edema following panretinal (scatter) photocoagulation given in 1 or 4 sittings," Archives of Ophthalmology, vol. 127, no. 2, pp. 132-140, 2009.

[23] S. B. Lee, Y. J. Yun, S. H. Kim, and J. Y. Kim, "Changes in macular thickness after panretinal photocoagulation in patients with severe diabetic retinopathy and no macular edema," Retina, vol. 30, no. 5, pp. 756-760, 2010.

[24] M. Shimura, K. Yasuda, T. Nakazawa, T. Kano, S. Ohta, and M. Tamai, "Quantifying alterations of macular thickness before and after panretinal photocoagulation in patients with severe diabetic retinopathy and good vision," Ophthalmology, vol. 110, no. 12, pp. 2386-2394, 2003.

[25] M. M. K. Muqit, G. R. Marcellino, J. C. B. Gray et al., "Pain responses of Pascal $20 \mathrm{~ms}$ multi-spot and $100 \mathrm{~ms}$ singlespot panretinal photocoagulation: Manchester Pascal Study, MAPASS report 2," British Journal of Ophthalmology, vol. 94, no. 11, pp. 1493-1498, 2010.

[26] A. Mirshahi, A. Lashay, M. Roozbahani et al., "Pain score of patients undergoing single spot, short pulse laser versus conventional laser for diabetic retinopathy," Graefe's Archive for Clinical and Experimental Ophthalmology, vol. 251, no. 4, pp. 1103-1107, 2013.

[27] A. V. Chappelow, K. Tan, N. K. Waheed, and P. K. Kaiser, "Panretinal photocoagulation for proliferative diabetic retinopathy: pattern scan laser versus argon laser," American Journal of Ophthalmology, vol. 153, no. 1, pp. 137.e2-142.e2, 2012.

[28] J.-H. Oh, S.-W. Kim, S.-S. Kwon, and K. Huh, "The change of macular thickness following single-session pattern scan laser panretinal photocoagulation for diabetic retinopathy," Graefe's Archive for Clinical and Experimental Ophthalmology, vol. 253, no. 1, pp. 57-63, 2015.

[29] O. Comyn, L. Z. Heng, F. Ikeji et al., "Repeatability of Spectralis OCT measurements of macular thickness and volume in diabetic macular edema," Investigative Ophthalmology and Visual Science, vol. 53, no. 12, pp. 7754-7759, 2012.

[30] T. Fiore, S. Androudi, B. Iaccheri et al., "Repeatability and reproducibility of retinal thickness measurements in diabetic patients with spectral domain optical coherence tomography," Current Eye Research, vol. 38, no. 6, pp. 674-679, 2013.

[31] E. H. Sohn, J. J. Chen, K. Lee, M. Niemeijer, M. Sonka, and M. D. Abràmoff, "Reproducibility of diabetic macular edema estimates from SD-OCT is affected by the choice of image analysis algorithm," Investigative Ophthalmology \& Visual Science, vol. 54, no. 6, pp. 4184-4188, 2013. 


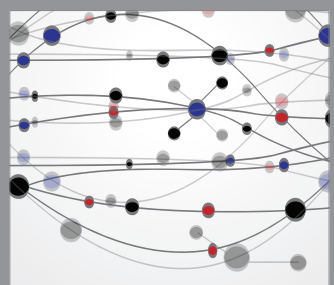

The Scientific World Journal
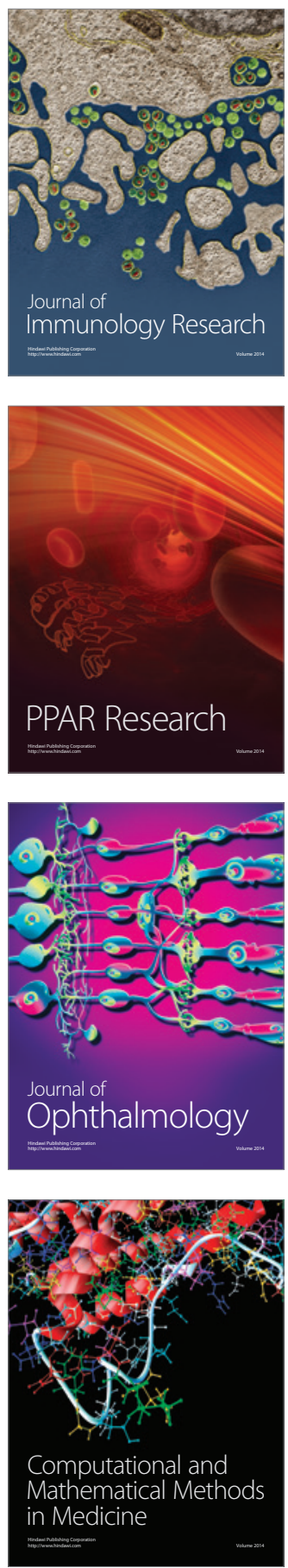

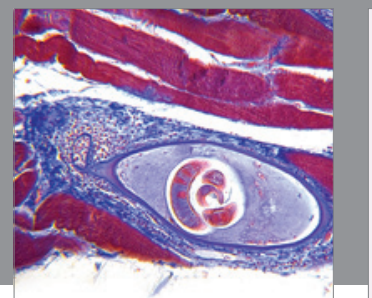

Gastroenterology

Research and Practice
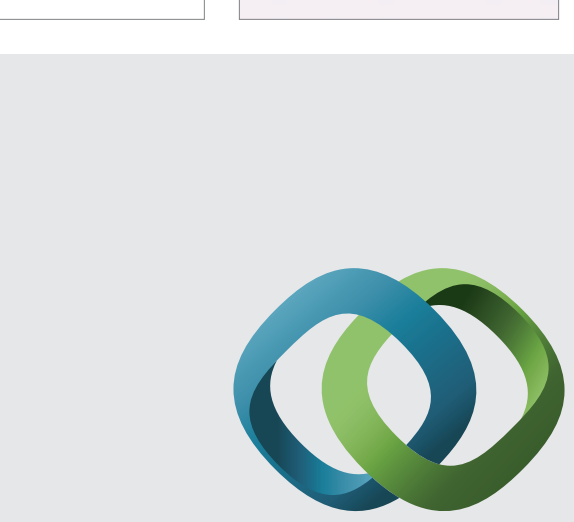

\section{Hindawi}

Submit your manuscripts at

http://www.hindawi.com

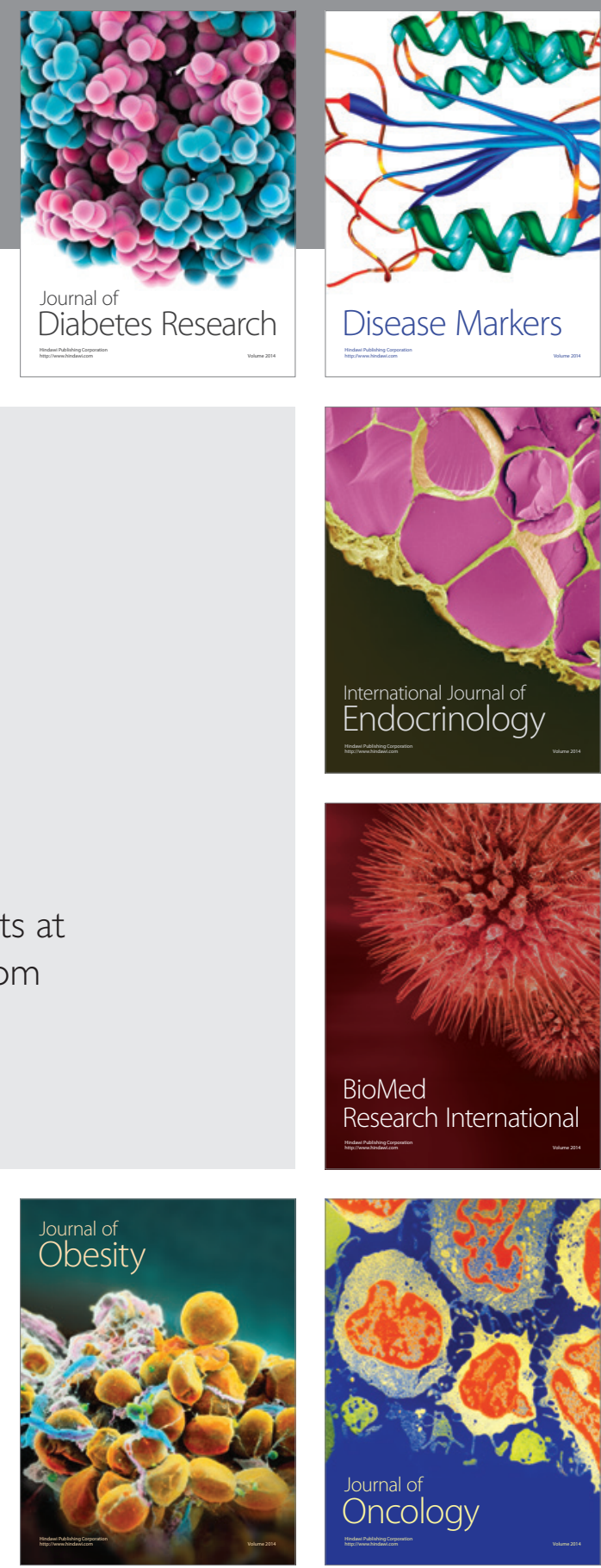

Disease Markers
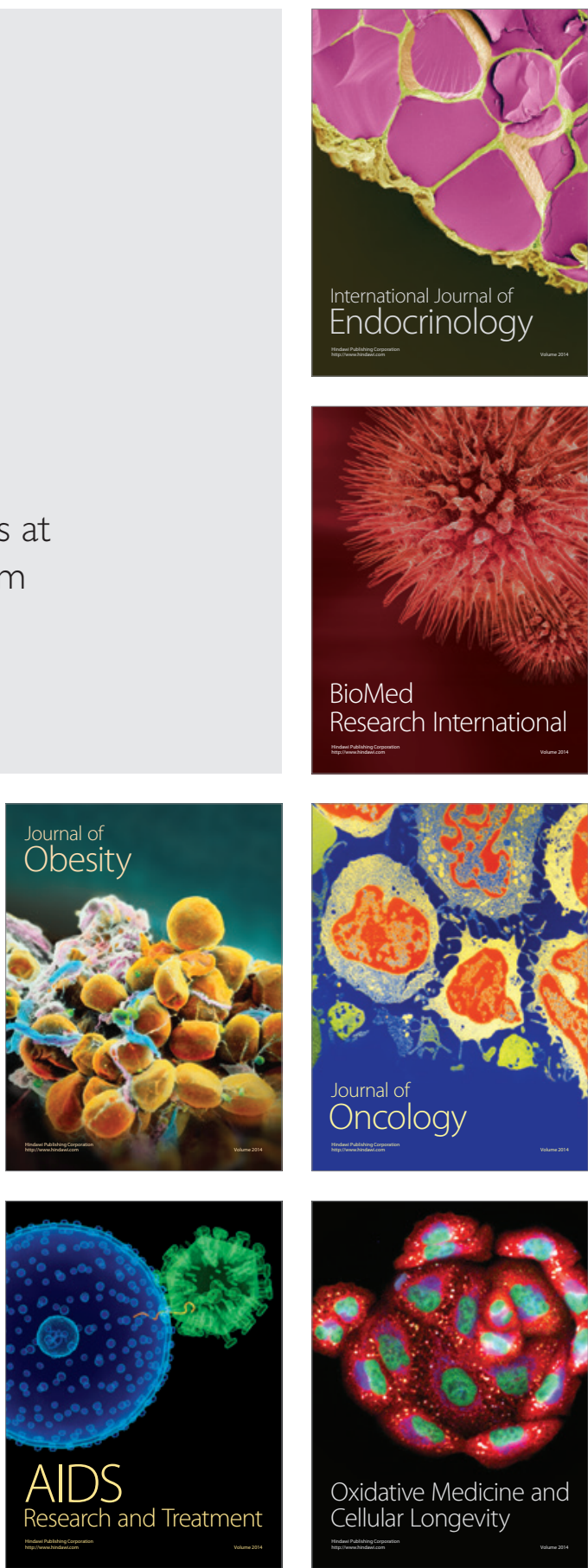